\title{
A Content-based Image Retrieval System Based on Polar Raster Edge Sampling Signature
}

\author{
Santhosh P. Mathew ${ }^{1}$, Valentina E. Balas ${ }^{2}$, Zachariah K. P. ${ }^{1}$, \\ Philip Samuel $^{3}$ \\ ${ }^{1}$ Department of Computer Science, Saintgits College of Engineering, Kerala, \\ India; santhosh.mathew@ saintgits.org; zacharia.kp@saintgits.org \\ ${ }^{2}$ Department of Automatics and Applied Software, Aurel Vlaicu University of \\ Arad, Romania; valentina.balas@uav.ro \\ ${ }^{3}$ Department of Information Technology, Cochin University of Science and \\ Technology, Cochin, Kerala, India; philips@ cusat.ac.in
}

\begin{abstract}
Content Based Image Retrieval (CBIR) is used to effectively retrieve required images from fairly large databases. CBIR extracts images that are relevant to the given query image, based on the features extracted from the contents of the image. Most of the CBIR systems available in the literature are not rotation and scale invariant. Retrieval efficiency is also poor. In this paper, shape features are extracted from the database images and the same are polar raster scanned into specified intervals in both radius and angle, using the proposed Polar Raster Edge Sampling Signature (PRESS) algorithm. Counts of edge points lying in these bins are stored in the feature library. When a query image passed on to the system, the features are extracted in the similar fashion. Subsequently, similarity measure is performed between the query image features and the database image features based on Euclidian Distance similarity measure and the database images that are relevant to the given query image are retrieved. PRESS algorithm has been successfully implemented and tested in a CBIR System developed by us. This technique preserves rotation and scale invariance. It is evaluated by querying different images. The retrieval efficiency is also evaluated by determining precision-recall values for the retrieval results.
\end{abstract}

Keywords: image retrieval; polar raster edge sampling signature; image segmentation; feature extraction; K-means clustering; Canny algorithm

\section{Introduction}

Content based Image Retrieval system uses content for the retrieval process. It is not easy to develop such a retrieval system because of the complexity involved in real world images that contain complex objects and detailed color information [1]. 
An image retrieval system is a computer system for browsing, searching and retrieving images from a large database of digital images. Some of the important indications that are used to extract information from the images are Color, Shape and texture. Color histograms are widely used in content based image retrieval [2]. Though color and texture contain key information, it is possible that different images with similar color histograms represent very different things. Hence shapedescribing features are to be used in an efficient content-based image retrieval system. Although much research has taken place in connection with shape description to identify the right kind of shape feature, there are no direct answers yet [3]. Most of the traditional methods of image retrieval use some method of adding metadata. Captioning, Keywords or Descriptions are added to the images so that retrieval can be performed based on the annotation words.

Image retrieval has been an area of interest for researchers during the past few decades. Database Management and Computer Vision are two main research groups who study image retrieval from different viewpoints. Database Management group primarily looks at text-based approaches and the Computer Vision group looks at visual-based approaches [4]. There is a basic difference between content-based and text-based retrieval systems. Text-based systems are depended on human interaction. Humans normally use keywords, text descriptors and similar high level features to interpret images [5]. Manual textual annotation was used initially to retrieve images. But this was observed to be a very difficult task, primarily because of the limitation in the interpretation of what we see. This resulted in the image contents like color, shape and texture gaining greater importance [6]. Also, large amount of manual effort was required in developing the annotations and in addressing the differences in interpretation of image contents and the lack of uniformity of the keyword assignments among various indexers. The keyword annotation approach becomes impractical as the size of the image database increases. To overcome these difficulties another mechanism, Content Based Image Retrieval, is used [4].

One of the important basic features in content-based image retrieval is Shape. Shape based representations are broadly divided into two: Region based and Contour based [7]. Moment descriptors - Geometrical moments, Zernike moments and Legendre moments are normally used in the case of Region based representation [8], [9]. Contour based systems normally use the boundary of the objects for representation and retrieval. There are different effective algorithms to extract the edges. Since images are usually distinguishable by their contours, this approach gives better results [11].

CBIR systems retrieve images that match the extracted content features of the query image. Identifying a suitable feature set that would ensure retrieval efficiency has been a challenge in Content Based Image Retrieval. Rotation and Scale invariance too have not been addressed effectively. The proposed CBIR system retrieves a specified number of images that match the query image. The best match will be displayed first and it also preserves Rotation and Scale Invariance. 
The remaining parts of the paper are arranged as follows: In Section 2, the background of the work is described. Section 3 briefs the Content based image retrieval with proposed Polar Shape Signature extraction, Feature calculation and Image retrieval process. Experimental results and analysis of the proposed technique are discussed in Section 4. Finally, concluding remarks are provided in Section 5.

\section{Background of the Work}

A brief discussion of some relevant works from the available literature in the area of shape based Content Based Image Retrieval is presented in this section.

Number of trademark images around the world has increased rapidly. Trademark image retrieval (TIR) has been researched upon to ensure that new trademarks are not a replica of the trademark images that are stored in the trademark registration system. Chia-Hung Wei et al. [8] suggested a content-based trademark retrieval system using feature sets that could describe global shapes and local aspects of the trademarks. Color, texture and shape information are the common image descriptors in content based image retrieval systems. Xiang-Yang, Wang et al. [10] proposed a new combination color image retrieval scheme, making use of all these descriptors.

Shape is an important visual feature in CBIR. Shape descriptors are of two types i.e., contour-based and region-based. Contour-based shape descriptors use only the boundary information by neglecting the content within the shape, while regionbased shape descriptors concentrate on the contents within the shape.

Zang and Melissa [12] proposed a method for shape representation and retrieval, taking into account the regional properties. They proposed an idea that is similar to normal raster sampling. But, instead of using the normal square grid on a shape image, a circular sampling of concentric circles and radial lines is used at the center of the shape. The binary value of the shape is sampled at the intersections of the circles and radial lines. Santhosh P. Mathew et al [13] have also tried to implement similar concept for a CBIR system. The drawback of this technique is that the shape points on the intersection are only considered. PRESS proposed by us forms an effective shape signature by taking into account all the edge points, by collecting them in specified number of radial and angular bins. Scaling and rotational invariance are also preserved in the CBIR system implemented using PRESS. 


\section{Content-based Image Retrieval Based on Polar Raster Edge Sampling Signature (PRESS)}

The proposed effective Content Based Image Retrieval system comprises of the processes such as Image Segmentation, Feature Extraction and Image retrieval based on the query image. Here the CBIR is based on the extracted shape signature. The shape signature extraction is as follows. Initially, the image is segmented based on color, using the $\mathrm{K}-$ Means clustering algorithm. Canny algorithm is employed to detect the edges. The strong edges and the connected edges are identified using various techniques like double thresholding and edge tracking. The edge data in the boundary and the region are polar raster scanned in both radius and angle. Numbers of edge points identified are stored in the feature library for all the database images. When an image is queried, the system extracts shape feature for the image in the same way and then computes the similarity measure between the features of the query image and the feature existing in the feature database based on the Euclidean Distance method. Minimum distance indicates the closest match and specified number of best matched images are extracted. The proposed method is detailed in the following sections.

\subsection{Extraction of Shape Features}

Initially, the image in RGB color space is converted to gray scale. We make use of the K-means clustering algorithm to segment image for further processing [14] [15]. After the k means algorithm is applied, the canny algorithm is used for the detection of different edges present in all the clustered sets of the image. Then we get the different shapes that are present in the image and the features of the shaped content are extracted by employing the proposed PRESS (Polar Raster Edge Sampling Signature) algorithm. Edge data of the shape is polar raster scanned or binned into 10 intervals in both radius and angle. Counts of the edge points lying in these bins are found and stored in two vectors $\mathbf{r}$ and $\mathbf{t}$. The entire shape feature extraction process can be represented in the following pseudo code.

In this pseudo code the proposed PRESS algorithm is used for extracting the shape features of the image. The approach is that the final edge components extracted are polar raster scanned or binned into ten intervals in the Radius and the Angle. Count of edge points lying in these bins are found and stored as two vectors $\mathbf{r}$ and t. It is further normalized for sum of counts. The same process is repeated for all the images in the database and the $r$ and $t$ values are saved in the feature database. 
$B W \leftarrow$ edge $($ mask, 'canny'); $\quad / /$ Finding the edges of the image.

$[\operatorname{imx}$, imy $] \leftarrow \operatorname{size}(B W)$;

$B \leftarrow \operatorname{conv} 2(B W, m s k) ; \quad / /$ Smoothing the image

$[x, y] \leftarrow$ find $(B==1)$;

$p=[x, y]$;

$[q r, q t]=$ polartransform $(p)$;

get count of edge points;

store radius bin count in vector $r$,

store angle bin count in vector $t$;

//PRESS is used to extract shape features

normalize for sum of counts;

repeat for all images in the $D B$;

save them in feature $D B$;

Figure 1

Pseudo code for shape feature extraction process
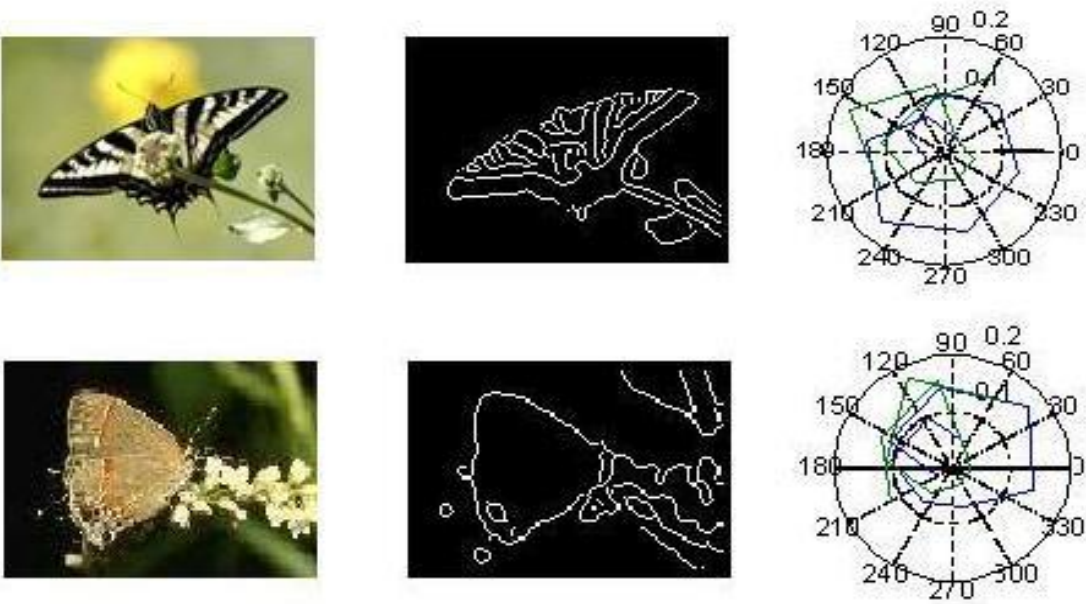

Figure 2

PRESS algorithm applied on the edges 


\subsection{Retrieval Process}

Image features are extracted first and the appropriate match of the query image is retrieved from the database. The similarity measure between shape feature of query image $Q$ and the features of each image in the database is calculated using Euclidean Distance (ED). The feature of query image is represented as $Q_{f}=[r, t]$.

$$
d_{i}=\left\|Q_{f}-F_{i}\right\|
$$

ED between $r$ vectors of query and DB is found. Minimum ED between $t$ vector of query image and circularly shifted t vector of DB image is found to account for possible rotation of query image. RMS of the two EDs is used as the distance measure between shape features. Minimum distance is the closest match. On the basis of the above similarity measure, a specified number of best matched images from the DB that are similar to the query image are retrieved.

\section{Results and Discussion}

We have implemented this CBIR system in MATLAB 7.10. Test images were taken from the database generated by Wang containing many images stored in the JPEG format [16]. The system extracts the shape feature for the query image and then computes the similarity measure between that and the shape features of all images existing in the database. This results in a predefined number of database images similar to the query image being retrieved.

The query image is first preprocessed to normalize the intensity levels of the input image. The output objects extracted from the input query image after the preprocessing stage is given in Row 2 of Fig. 3. Since the mean filter acts only on a single color channel, the RGB image is first converted to gray scale image, using Craig's formula. K-means clustering algorithm $(\mathrm{k}=4)$ is used on the gray scale image for the segmentation of the image. Before applying the K-means clustering, the image in the form of $2 \mathrm{D}$ vector is rescaled to a $1 \mathrm{D}$ vector. The output image of the clustering process is shown in the Row 3 of Fig. 3. After the clustering process using K-Means algorithm, the 1D image is again converted back into $2 \mathrm{D}$ image format. Subsequently, Canny algorithm is applied to detect the edges. Edges are present in all the clustered sets of the image and Canny algorithm identifies them. The various steps involved are smoothing, finding gradients, non-maximum suppression, double thresholding and edge tracking by hysteresis. The detected edges are shown in the Row 4 of Fig. 4. 

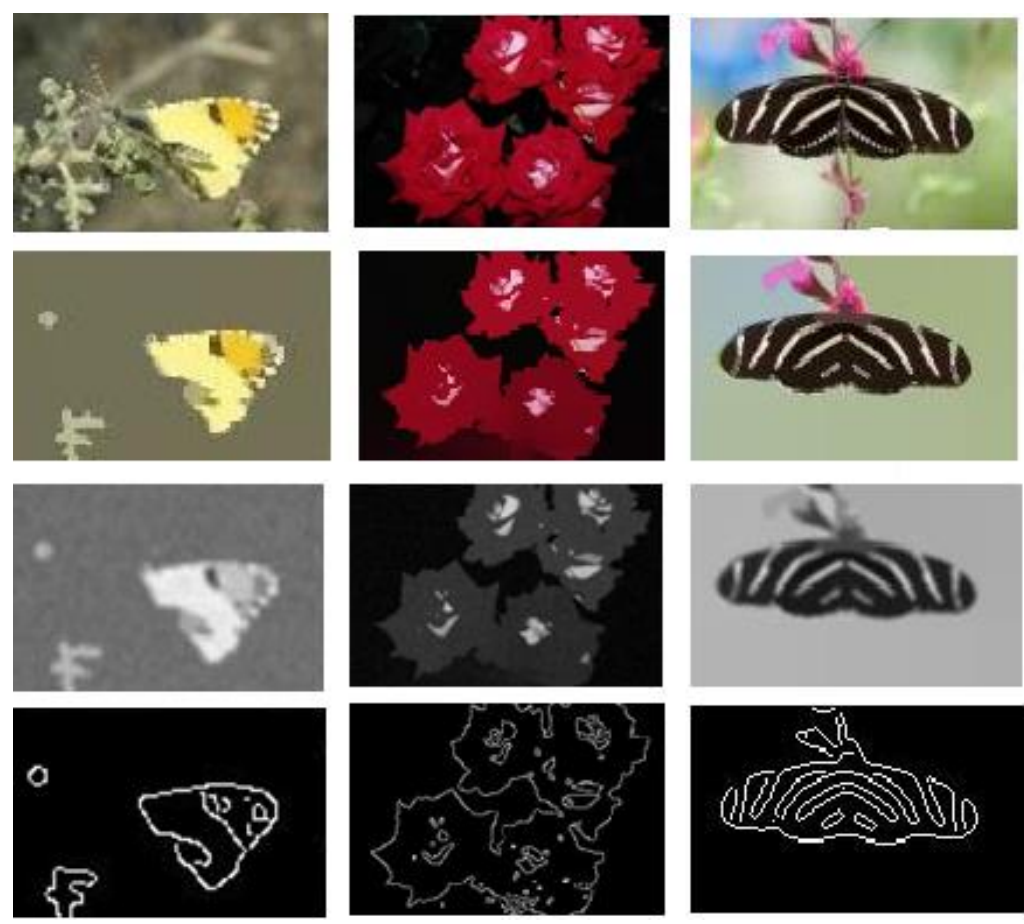

Figure 3

Row 1 - Original images, Row 2 - Objects extracted from the original images

Row 3 - Clustered gray scale images, Row 4 - Edges detected by Canny algorithm

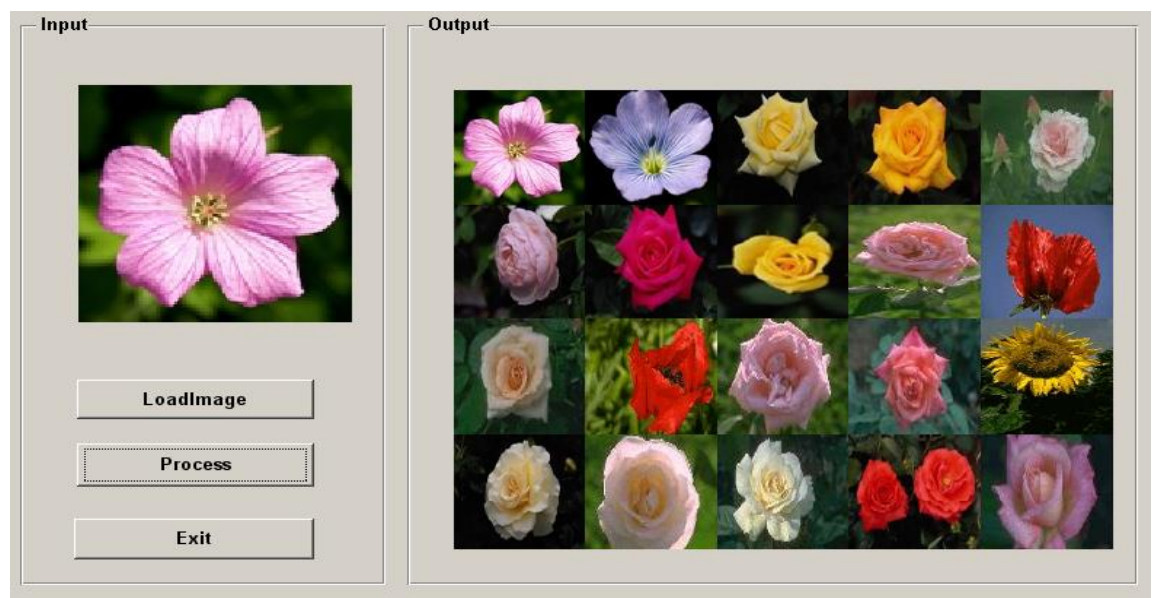

Figure 4

Sample output - Query Image and Retrieved images 
Suppressing and smoothing are done on these edges to eliminate unused connected components and those edges that are not connected to strong ones. Thus the clear edges of all the segments are obtained. PRESS algorithm proposed by us is employed on the final edge components to extract the shape features. Query image shape features are matched with shape features in the DB by using RMS values of the EDs of $r$ vectors and $t$ vectors. Minimum distance is the closest match.
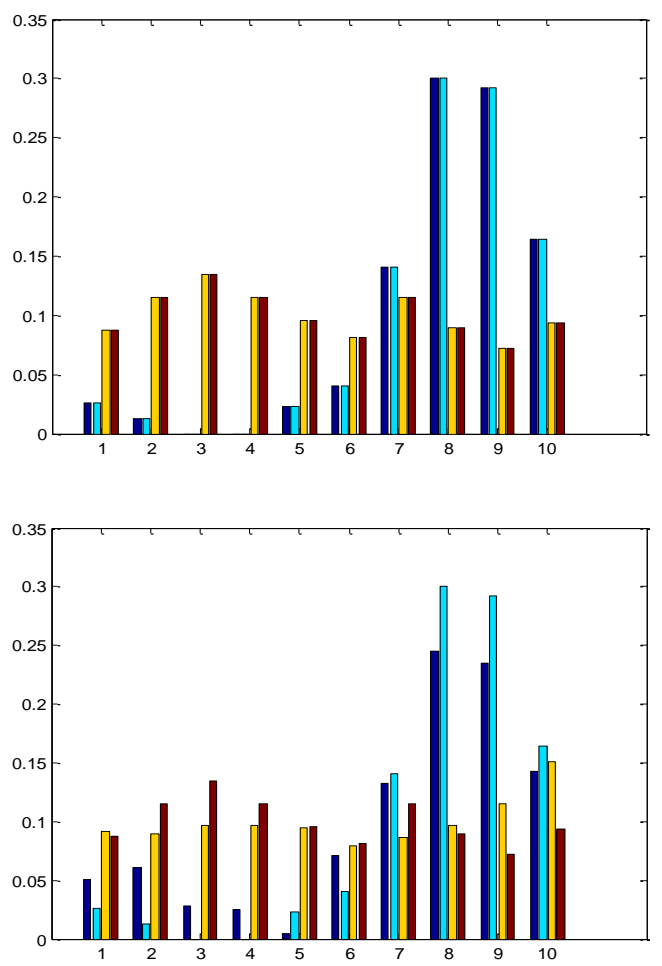

Figure 5

Bar graph indicating the exact match of the Radius bin count (rr), Theta bin count (rt), with that of the query image (qr, qt) - for the best match. Bar graph for the next best match is also shown along side.

Thus the images corresponding to the input query image are retrieved from the database. Some of the retrieved images that correspond to the input query image are shown in Fig. 4. Bar graph of the $r$ and t values of the query and retrieved images are given in Fig. 5. The PRESS technique successfully matches the query image exactly with that in the database and can in no way miss it. Rotational Invariance and Scale Invariance were also tested with rotated and scaled images. At all instances, all the rotated and scaled images were retrieved without any problems. Sample outputs for Rotational and Scale Invariance are given in Fig. 6 and Fig. 7. 


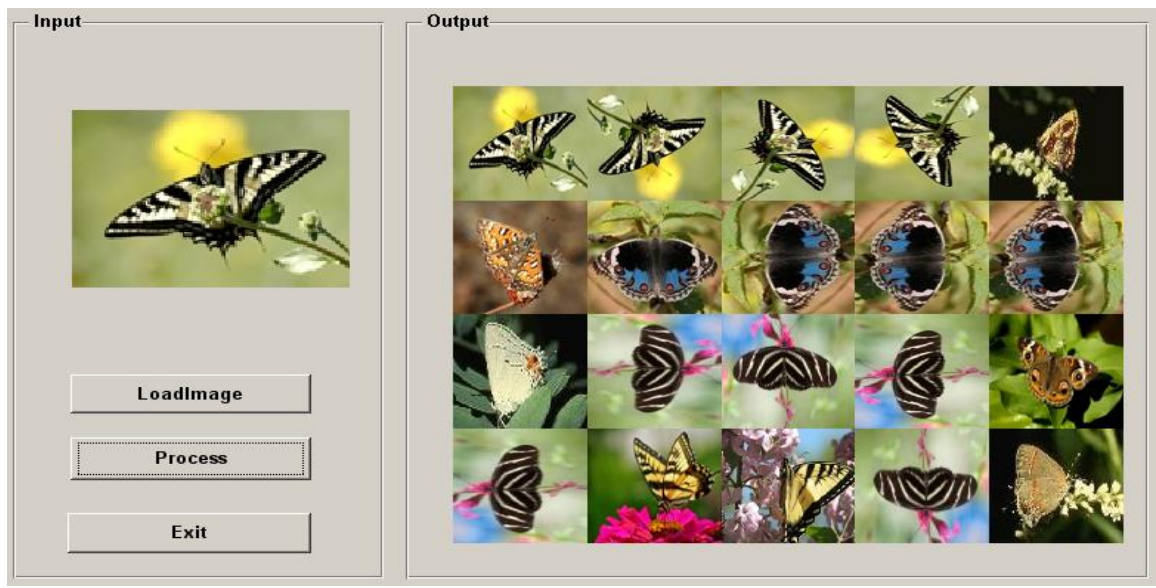

Figure 6

Rotational Invariance - Query Image and Retrieved images - that were rotated in 90, 180, 270 and 360 degrees

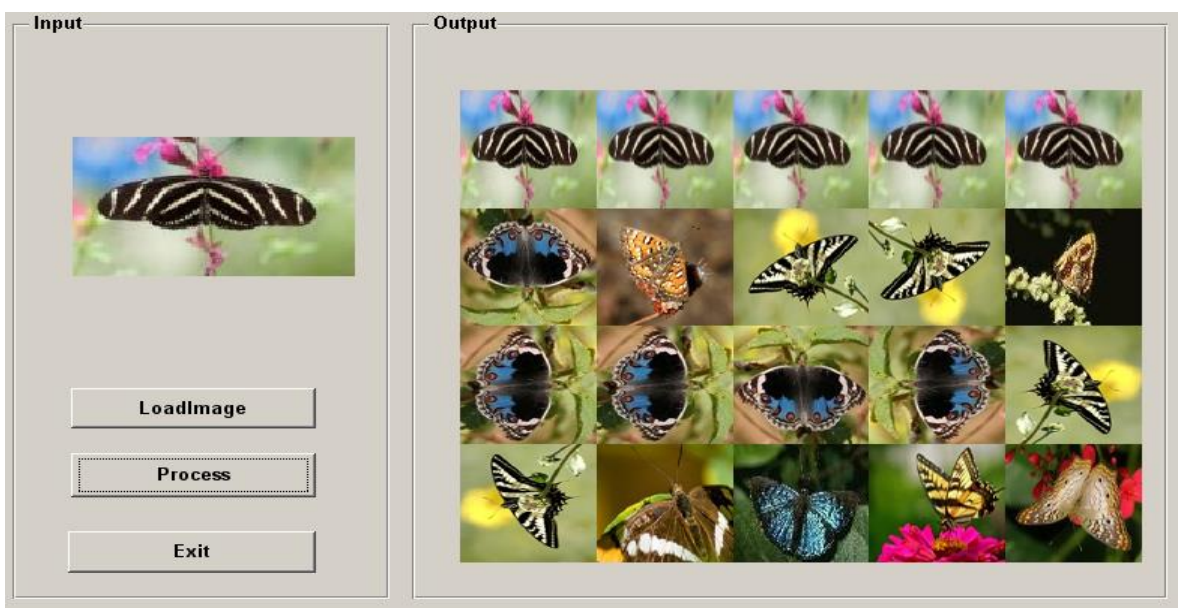

Figure 7

Scale Invariance - Query image $(143 \times 82)$ and Retrieved images - that were scaled to $157 \times 90,172 \times 98$, $200 \times 115$ and $129 \times 74$

\section{Comparative Analysis}

Precision and recall values are used to analyze the performance. Precision is the fraction of retrieved images that are relevant to the query image. Recall is the fraction of relevant images that are retrieved from the database. We obtain a measure of relevance, from these values.

$$
\text { precision }=\frac{\text { Count of retrieved images relevant to the query image }}{\text { Total count of images retrieved }}
$$




$$
\text { recall }=\frac{\text { Count of retrieved images relevant to the query image }}{\text { Total count of relevant images in the database }}
$$

Using Eq. (2) and Eq. (3), the precision and recall values for the query image are calculated for the proposed method and for some of the existing methods. The values obtained from the calculation are given in Table I. The precision values are compared with some of the existing methods and plotted in Fig. 8.

Table 1

Precision (P) and Recall (R) Statistics for the proposed PRESS (Polar Raster Edge Sampling

Signature) Method on the best 15, 20 and 40 retrieved images

\begin{tabular}{|c|c|c|c|c|c|c|}
\hline \multirow{2}{*}{ Query } & \multicolumn{2}{|c|}{$\mathrm{N}=15$} & \multicolumn{2}{c|}{$\mathrm{N}=20$} & \multicolumn{2}{c|}{$\mathrm{N}=40$} \\
\cline { 2 - 7 } & $\mathrm{P}$ & $\mathrm{R}$ & $\mathrm{P}$ & $\mathrm{R}$ & $\mathrm{P}$ & $\mathrm{R}$ \\
\hline 1 & 0.80 & 0.28 & 0.75 & 0.35 & 0.73 & 0.67 \\
\hline 2 & 1 & 0.35 & 0.95 & 0.44 & 0.85 & 0.79 \\
\hline 3 & 1 & 0.35 & 0.95 & 0.44 & 0.83 & 0.76 \\
\hline 4 & 0.80 & 0.28 & 0.75 & 0.35 & 0.70 & 0.65 \\
\hline 5 & 1 & 0.35 & 0.95 & 0.44 & 0.93 & 0.86 \\
\hline 6 & 0.93 & 0.33 & 0.85 & 0.40 & 0.80 & 0.74 \\
\hline 7 & 0.80 & 0.28 & 0.75 & 0.35 & 0.73 & 0.67 \\
\hline 8 & 0.80 & 0.28 & 0.75 & 0.35 & 0.70 & 0.65 \\
\hline 9 & 0.93 & 0.33 & 0.80 & 0.37 & 0.78 & 0.72 \\
\hline 10 & 0.80 & 0.28 & 0.75 & 0.35 & 0.70 & 0.65 \\
\hline
\end{tabular}

Fig. 8 shows the graph for the comparative analysis between the proposed Polar Raster Edge Sampling Signature (PRESS) method and existing methods like CSS (Curvature Scale Space), ZMD (Zernike Moment Descriptor), GFD (Geometric Fourier Descriptor) and MSFD (Multiple Shape Feature Descriptor) on 5 queries for a set of best 20 images retrieved. The data for other methods are from [10], [7].

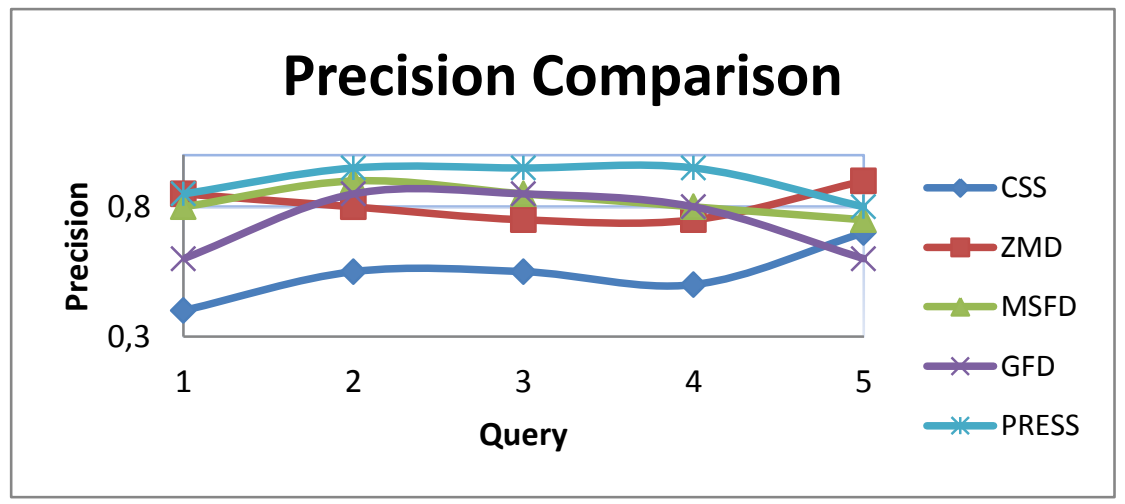

Figure 8

Comparison of Precision 


\section{Conclusion}

We have proposed a CBIR system based on Shape Signature to retrieve relevant matches from the database for the query image. When an image is queried, shape feature for the image is extracted and then the similarity measure between the features of the query image and the feature existing in the feature database are calculated based on the Euclidean Distance method. Image segmentation is done using K-means clustering algorithm, which groups the image pixels under color. Canny algorithm is then used to extract edges. Proposed PRESS algorithm has been successfully applied on the edge components to extract shape features. The same is tested in a CBIR System developed by us. This technique preserves rotation and scale invariance. It is evaluated by querying different images. PrecisionRecall values are used for the comparison of retrieval results. The implementation results illustrates that this novel image retrieval process effectively retrieves the images that are very close to the query image from the database. Precision - Recall table and Precision comparison plot with existing CBIR techniques prove the effectiveness of the system.

\section{References}

[1] A. W. M. Smeulders, M. Worring, S. Santini, A. Gupta and R. Jain: Content-Based Image Retrieval at the End of the Early Years, IEEE Transactions on Pattern Analysis and Machine Intelligence, Vol. 22, No. 12, Dec. 2000, pp. 1349-1379

[2] S. Belongie, C. Carson, H. Greenspan and J. Malik: Color and TextureBased Segmentation using EM and its Application to Content-based Image Retrieval, In Proc. of the Sixth International Conference on Computer Vision, Vol. 10, Jan. 1998, pp. 675-682

[3] J. Laaksonen, E. Oja and S. Brandt: Statistical Shape Features in Contentbased Image Retrieval, In Proc. of the $15^{\text {th }}$ International Conference on Pattern Recognition, Vol. 2, Sep. 2000, pp. 1062-1065

[4] Y. Rui and T. S. Huang: Image Retrieval: Current Techniques, Promising Directions, and Open Issues, Journal of Visual Communication and Image Representation, Vol. 10, Jan. 1999, pp. 39-62

[5] Y. Liu, D. Zhang, G. Lu and W.-Y. Ma: A Survey of Content-based Image Retrieval with High-level Semantics, Journal of Pattern Recognition, Vol. 40, No. 1, Jan. 2007, pp. 262-282

[6] A. Jain, R. Muthuganapathy and K. Ramani: Content-based Image Retrieval Using Shape and Depth from an Engineering Database, In Proc. of the Third International Conference on Advances in Visual Computing, Vol. 2, 2007, pp. 255-264

[7] D. Zhang and G. Lu: A Comparative Study of Curvature Scale Space and Fourier Descriptors for Shape-based Image Retrieval, Journal of Visual 
Communication and Image Representation, Vol. 14, No. 1, Mar. 2003, pp. $39-57$

[8] C.-H. Wei, Y. Li, W. Y. Chau and C.-T. Li: Trademark Image Retrieval Using Synthetic Features for Describing Global Shape and Interior Structure, Journal of Pattern Recognition, Vol. 42, No. 3, Mar. 2009, pp. 386-394

[9] D. Zhang and G. Lu: Shape-based Image Retrieval using Generic Fourier Descriptor, Journal of Signal Processing: Image Communication, Vol. 17, No. 10, Nov. 2002, pp. 825-848

[10] X.-Y. Wang, Y.-J. Yu and H.-Y. Yang: An Effective Image Retrieval Scheme using Color, Texture and Shape Features, Journal of Computer Standards \& Interfaces, Vol. 33, Mar. 2010, pp. 59-68

[11] Ch. S. Rao, S. S. Kumar and B. C. Mohan: Content-based Image Retrieval Using Exact Lengendre Moments and Support Vector Machine, The International Journal of Multimedia \& Its Applications, Vol. 2, No. 2, May 2010, pp. 69- 79

[12] D. Zhang and M. C. Yi Lim: An Efficient and Robust Technique for Region-based Shape Representation and Retrieval, $6^{\text {th }}$ IEEE/ACIS International Conference on Computer and Information Science (ICIS 2007) pp. 801-806

[13] S. P. Mathew and P. Samuel: A Novel Image Retrieval System using an Effective Region-based Shape Representation Technique, International Journal of Image Processing (IJIP), Vol. 4, No. 5, Dec. 2010, pp. 509-517

[14] K. S. Ravichandran and B. Ananthi, Color Skin Segmentation Using KMeans Cluster, International Journal of Computational and Applied Mathematics, Vol. 4, No. 2, 2009, pp. 153-157

[15] Szabolcs Sergyán: A New Approach of Face Detection-based Classification of Image Databases, Acta Polytechnica Hungarica, Journal of Applied Sciences, Vol. 6, No. 1, 2009, pp. 175-184

[16] R. Datta, D. Joshi, J. Li and J. Z. Wang: Image Retrieval: Ideas, Influences, and Trends of the New Age, ACM Computing Surveys, Vol. 40, No. 2, April 2008, Article 5 